\title{
TOXIC EFFECTS OF COPPER SULPHATE PENTAHYDRATE ON ANTIOXIDANT ENZYME ACTIVITIES AND LIPID PEROXIDATION OF FRESHWATER FISH CAPOETA UMBLA (HECKEL, 1843) TISSUES
}

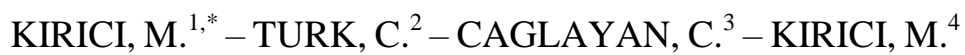 \\ ${ }^{1}$ Department of Fisheries, Faculty of Agriculture, Bingöl University \\ 12000-Bingöl, Turkey \\ ${ }^{2}$ Department of Pathology, Faculty of Veterinary Medicine, Bingol University \\ 12000-Bingöl, Turkey \\ ${ }^{3}$ Department of Biochemistry, Faculty of Veterinary, Bingöl University \\ 12000-Bingöl, Turkey \\ ${ }^{4}$ Department of Chemistry, Faculty of Arts and Science, Bingöl University \\ 12000-Bingöl, Turkey \\ *Corresponding author \\ e-mail: muammerkirici@hotmail.com \\ (phone: +90-536-891-7150; fax: +90-426-216-0029) \\ (Received $17^{\text {th }}$ Feb 2017; accepted $2^{\text {nd }}$ Jun 2017)
}

\begin{abstract}
Copper sulphate pentahydrate $\left(\mathrm{CuSO}_{4} .5 \mathrm{H}_{2} \mathrm{O}\right)$ is commonly used as a general biocide in the agricultural fields surrounding freshwater reservoirs. So the aim of the present study was to investigate the toxic effects of $\mathrm{CuSO}_{4} .5 \mathrm{H}_{2} \mathrm{O}$ by examining oxidative damage markers such as malondialdehyde (MDA) for lipid peroxidation and the antioxidant enzymatic defense system components (glutathione reductase (GR), glucose 6-phosphate dehydrogenase (G6PD), superoxide dismutase (SOD), glutathione peroxidase (GPx) and catalase (CAT)) in freshwater fish Capoeta umbla (Heckel, 1843) liver, kidney and gill. In this purpose, the $96 \mathrm{~h} \mathrm{LC}_{50}$ values (with $95 \%$ confidence limits) of $\mathrm{CuSO}_{4} .5 \mathrm{H}_{2} \mathrm{O}$ for $C$. umbla was estimated as a $1.478 \mathrm{mg} / \mathrm{L}$. Three different $\mathrm{CuSO}_{4} .5 \mathrm{H}_{2} \mathrm{O}$ sublethal doses $(0.185,0.370$ and $0.739 \mathrm{mg} / \mathrm{L})$ were administered to C. umbla at different time intervals (12, 24, 48, 72 and $96 \mathrm{~h})$. Antioxidant enzyme activities and MDA levels were measured spectrophotometrically. Liver, kidney and gills samples were collected at the end of the experiment, and analysed for their oxidant-antioxidant status, including MDA level, GR, G6PD, SOD, CAT and GPx activities. Results obtained showed that $\mathrm{CuSO}_{4} .5 \mathrm{H}_{2} \mathrm{O}$, significantly decreased $(\mathrm{P}<0.05)$ the GR, G6PD and GPx activities, but increased $(\mathrm{P}<0.05)$ the CAT, SOD activities and MDA levels of $C$. umbla liver, kidney and gill tissue in a dose-dependent manner. However, $\mathrm{CuSO}_{4} .5 \mathrm{H}_{2} \mathrm{O}$ exposure did not cause to changes significantly $(\mathrm{P}<0.05)$ in enzyme activities and MDA levels of $C$. umbla liver, kidney and gill tissue in a time-dependent manner.
\end{abstract}

Keywords: pesticide, freshwater fish, aquatic contamination, antioxidant enzymes, oxidative damage markers

\section{Introduction}

Chemical pollution in the environment by pesticides has been increasing due to their extensive usage in agriculture. Many of these compounds or their metabolites have shown toxic effects related to oxidative stress in the freshwater fauna, particularly fish. Oxidative stress occurs when the critical balance between oxidants and antioxidants is disrupted due to the depletion of antioxidants or excessive accumulation of the reactive oxygen species, or both, leading to damage (Kumar et al., 2011; Yonar and Sakin, 2011; 
Ural, 2013). Chemical pollution in the environment by pesticides are able to induce oxidative stress by accelerating the generation of highly reactive oxygen species (ROS), including superoxide radical $\left(\mathrm{O}_{2}^{-}\right)$, hydrogen peroxide $\left(\mathrm{H}_{2} \mathrm{O}_{2}\right)$, hydroxyl radicals $\left(\mathrm{OH}^{*}\right)$, and singlet oxygen species $\left({ }^{1} \mathrm{O}_{2}\right)$. If not detoxified, these ROS can oxidize proteins, lipids and nucleic acids, often leading to damage in different cellular targets or even cell death (Lushchak, 2011; Qu et al., 2014). Fish are particularly sensitive to water contamination and pollutants may impair many physiological and biochemical processes when assimilated by fish tissue (Durmaz et al., 2006). When abnormal or xenobiotic-induced ROS production exceeds the endogenous protection, damage to cellular components can be often observed. This process is known as oxidative stress (Oakes and Van der Kraak, 2003). The antioxidant defense system includes enzymes such as superoxide dismutase (SOD), glutathione peroxidase (GPx), catalase (CAT), glutathione S-transferase (GST) and other low molecular weight scavengers such as reduced glutathione (GSH) (Storey, 1996; Droge, 2002; Monteiro et al., 2009;).

Copper pollution appears in the aquatic environment from natural and anthropogenic sources such as mine washing, agricultural leaching and direct application as algaecide and molluscicide. Monitoring biochemical and histo-cytological changes in fish liver is a highly sensitive and accurate way to assess the effects of xenobiotic compounds in laboratory and field studies. Moreover, liver is known to be the primary organ for copper storage in fish (Trivedi et al., 2012). The gill is the first tissue contacting with the contaminants in the water. Due to its large surface area and the small diffusion distance between the water and blood, the gills are primarily affected by contaminants such as metals. In general, the gill cells respond rapidly to various chemicals to overcome physiological impairment or tissue damage, and chemicals may have a negative effect on the overall gill function, enhancing fish susceptibility to toxic compounds and potentially leading to fish mortality (Demir et al., 2016). The gill is the main place for copper uptake, and because it has a constant and direct contact with the external environment (Atabati et al., 2015).

The vertebrate kidney is the main organ involved in the maintenance of body fluid homeostasis. In teleosts, the kidney, together with the gills and intestine, are responsible for excretion and the maintenance of the homeostasis of the body fluids and, besides producing urine, acts as an excretory route for the metabolites of a variety of xenobiotics to, which the fish may be exposed. In fish as in higher vertebrates, the kidney performs an important function related to electrolyte and water balance and the maintenance of a stable internal environment. Following exposure of fish to toxic agents such pesticides, histological alterations in the fish kidney were found at the level of the tubular epithelium and glomerulus (Yancheva et al., 2016).

The copper sulphate is worldwide used as an algaecide and a fungicide in aquaculture and agriculture (Lasiene et al., 2016). For example, copper sulfate is an effective treatment for filamentous algae in shrimp ponds (Chen and Lin, 2001). In the aquaculture industry, copper sulfate is used as a therapeutic chemical for various ectoparasitic and bacterial infections. It is reducing the incidence of fish parasites such as protozoa, trematodes, and external fungi and bacteria. It also inhibits growth of bacteria such as Salmonella spp., Pasteurella spp., Vibrio spp., Streptococcus spp., Aeromonas spp., Pseudomonas spp. and Edwardsiella spp. (Heo, 1997; Park and Heo, 2009; Nouh and Selim, 2013; Lasiene et al., 2016).

Increasing agricultural production leads to increasing the number of freshwater systems being impacted by the contaminants present in wastewater releases. $\mathrm{CuSO}_{4}$ is 
used extensively as a fungicide in agriculture. High concentration of this fungicide was detected in some aquatic ecosystems found in freshwater and in ground water (Atabati et al., 2015). However, scientists had proven copper sulphate pentahydrate toxic rather than preservative to many of the ornamental and commercially high valued fishes (Trivedi et al., 2012). The mechanism of $\mathrm{CuSO}_{4} .5 \mathrm{H}_{2} \mathrm{O}$ is partially known and still detailed study is required to understand its potential to produce oxidative stress at different dosage on liver, kidney and gill of freshwater fish Capoeta umbla. Therefore, the aim of the present study was to examine the influence of $\mathrm{CuSO}_{4} .5 \mathrm{H}_{2} \mathrm{O}$ on antioxidant enzyme activities and lipid peroxidation of freshwater fish Capoeta umbla liver, kidney and gill tissues.

\section{Materials and Methods}

\section{Experimental animal and design}

Capoeta umbla (Heckel, 1843) (107 \pm 12 g) samples were caught in Murat River, Bingöl, Turkey. Murat River is one of the most important large and long (722 km) tributary of the Euphrates River in South East Anatolia of Turkey. The distribution area of Murat River is upper basins systems of the Euphrates and Tigris River (Koyun, 2011; Kirici et al., 2016). After capturing, fish were transferred to at the Aquarium Fish Rearing Facility of the Department of Fishery Science, Agricultural Faculty at Bingöl University and fish were held in a $600 \mathrm{~L}$ water valum fiber glass tank. Prior to the experiments, fish were acclimatized for at least $21 \mathrm{~d}$ in aquria containing $600 \mathrm{~L}$ tap water. During the acclimatization period, they were fed with commercial pellets at $2 \%$ body weight thrice a day. But they were not fed during the last $24 \mathrm{~h}$ before starting of the experiment and throughout the experiment. The water quality parameters were measured as dissolved oxygen $8.42 \pm 0.7 \mathrm{mg} / \mathrm{L}, \mathrm{pH} 7.8 \pm 0.6$, temperature $17 \pm 4^{\circ} \mathrm{C}$, alkalinity $142 \pm 12 \mathrm{mg} / \mathrm{L}$ and total hardness $175 \pm 21 \mathrm{mg} / \mathrm{L}$ as $\mathrm{CaCO}_{3}$.

In the experiments, $\mathrm{CuSO}_{4} .5 \mathrm{H}_{2} \mathrm{O}$ was used the toxic compound. It was administered at concentrations of $0.5,1,1.5,2$ and $3 \mathrm{mg} / \mathrm{L}$ to the aquarium. Groups of experimental animals, each consisting of 10 individuals were selected at random and placed into aerated tank. The effect of the compound was determined in a period of $96 \mathrm{~h}$. The dead fish were immediately removed from the aquarium. After the $96 \mathrm{~h}, \mathrm{LC}_{50}$ value was calculated by a computer program. After $\mathrm{LC}_{50}$ value was calculated, fish were divided into four groups each containing 7 fishes $(n=7)$. Group I was held in tap water as control and other groups were exposed to $12.5 \%, 25 \%$ and $50 \%$ of $96 \mathrm{~h} \mathrm{LC}_{50}(0.185$, 0.370 and 0.739 ) for 12, 24, 48, 72 and $96 \mathrm{~h}$. No fish died during this period. The use of the fish and the experimental protocol were approved by the Animal Experimentation Ethics Committee of the Bingöl University (Bingöl, Turkey).

\section{Preparation of the homogenate}

At the end of the experiment, the fish were anaesthetized in anaesthetic matter (75 ppm, benzocaine) and tissues were removed rapidly. All tissues washed with physiological saline $(0.9 \% \mathrm{NaCl})$ and were cut into small pieces by using a scalpel. Tissues were suspended in a $50 \mathrm{mM} \mathrm{KH}_{2} \mathrm{PO}_{4}$ buffer ( $\mathrm{pH} \mathrm{7.4)}$ and were homogenized by liquid nitrogen. Homogenate was centrifuged at $27.000 \mathrm{~g}$ for $60 \mathrm{~min}$, supernatant existing on the upper section was carefully taken with a dropper. Following this, the precipitated part was removed. Supernatant was used to determine enzyme activity (Beutler, 1971). 


\section{Determination of lipid peroxidation and enzyme activities}

The levels of MDA, as indices of the LPO in all tissues, were measured using the thiobarbituric acid reactions according to the method of Placer et al. (1966). G6PD activity was measured at $37^{\circ} \mathrm{C}$ and $340 \mathrm{~nm}$, in accordance with the Beutler (1971) method. This method is based on the fact that NADPH, which is formed as a result of reducing $\mathrm{NADP}^{+}$. GR activity was measured at $25^{\circ} \mathrm{C}$ by the modified method of Carlberg and Mannervik (1975). SOD was assayed by the method of Sun et al. (1988) at $560 \mathrm{~nm}$ and $20^{\circ} \mathrm{C}$. CAT activity was determined by measuring the decomposition of hydrogen peroxide at $20^{\circ} \mathrm{C}$ and $240 \mathrm{~nm}$, according to the method of Aebi (1983). GPx was determined according to the method of Beutler (1975) by following the rate of nicotinamide adenine dinucleotide phosphate (NADPH) oxidation at $340 \mathrm{~nm}$.

\section{Statistical analysis}

Data were expresses as the mean \pm standard error (SE). The data were subjected to One-way ANOVA and Duncan's test was used to determine the significant difference between the control and experimental groups using the SPSS 17.0 computer program. A value of $\mathrm{p}<0.05$ was considered statistically significant.

\section{Results}

In this study, the calculated $96 \mathrm{~h}$ acute $\mathrm{LC}_{50}$ values (with $95 \%$ confidence limits) of $\mathrm{CuSO}_{4} .5 \mathrm{H}_{2} \mathrm{O}$, using a static bioassay system to $C$. umbla individuals $(\mathrm{n}=7)$ was 1.478 $\mathrm{mg} / \mathrm{L}$ by the use of Probit Analysis (Finney, 1971).

The levels of MDA in gill, kidney and liver tissues are shown in Table 1. The exposure to $\mathrm{CuSO}_{4} .5 \mathrm{H}_{2} \mathrm{O}$ caused a significant increase in levels of MDA in all tissues of $\mathrm{CuSO}_{4} .5 \mathrm{H}_{2} \mathrm{O}$ group in a dose-dependent manner. But no statistically significant change was observed in a time-dependent manner $(\mathrm{p}<0.05)$.

Table 1. Effect of $\mathrm{CuSO}_{4} .5 \mathrm{H}_{2} \mathrm{O}$ on $\mathrm{MDA}$ in gill, kidney and liver of $\mathrm{C}$. umbla (nm/mg protein)

\begin{tabular}{|c|c|c|c|c|c|c|}
\hline \multirow{2}{*}{ Indices } & \multirow{2}{*}{ Groups (mg/L) } & \multicolumn{5}{|c|}{ Times (hours) } \\
\hline & & 12 & 24 & 48 & 72 & 96 \\
\hline \multirow[t]{4}{*}{ Gill } & Control & $5.58 \pm 0.41$ & $5.46 \pm 0.43$ & $5.39 \pm 0.63$ & $5.32 \pm 0.78$ & $5.14 \pm 0.25$ \\
\hline & 0.185 & $5.91 \pm 0.60^{\mathrm{a}}$ & $6.47 \pm 1.62^{\mathrm{a}}$ & $7.88 \pm 0.22^{\mathrm{a}}$ & $7.91 \pm 1.12^{\mathrm{a}}$ & $7.98 \pm 1.75^{\mathrm{a}}$ \\
\hline & 0.370 & $6.07 \pm 0.97^{\mathrm{a}}$ & $7.16 \pm 1.49^{\mathrm{a}}$ & $7.42 \pm 1.19^{\mathrm{a}}$ & $8.71 \pm 1.21^{\mathrm{a}}$ & $9.06 \pm 0.65^{\mathrm{a}}$ \\
\hline & 0.739 & $7.23 \pm 1.85^{\mathrm{b}}$ & $9.11 \pm 1.39^{\mathrm{b}}$ & $9.42 \pm 1.32^{\mathrm{b}}$ & $10.57 \pm 1.16^{\mathrm{b}}$ & $10.81 \pm 0.99^{\mathrm{b}}$ \\
\hline \multirow[t]{4}{*}{ Kidney } & Control & $7.64 \pm 0.54$ & $7.82 \pm 0.74$ & $8.02 \pm 0.29$ & $7.98 \pm 0.87$ & $7.69 \pm 1.05$ \\
\hline & 0.185 & $7.77 \pm 1.76^{\mathrm{a}}$ & $8.97 \pm 1.10^{\mathrm{a}}$ & $8.36 \pm 1.71^{\mathrm{a}}$ & $8.32 \pm 1.42^{\mathrm{a}}$ & $9.92 \pm 1.77^{\mathrm{a}}$ \\
\hline & 0.370 & $8.40 \pm 1.88^{\mathrm{a}}$ & $9.01 \pm 1.41^{\mathrm{a}}$ & $10.591 .59^{\mathrm{a}}$ & $10.91 \pm 2.33^{\mathrm{a}}$ & $13.92 \pm 0.85^{\mathrm{a}}$ \\
\hline & 0.739 & $10.21 \pm 2.74^{b}$ & $11.06 \pm 1.94^{\mathrm{b}}$ & $11.93 \pm 1.03^{\mathrm{b}}$ & $12.96 \pm 1.55^{\mathrm{b}}$ & $15.31 \pm 1.02^{b}$ \\
\hline \multirow[t]{4}{*}{ Liver } & Control & $5.41 \pm 0.62$ & $5.57 \pm 0.20$ & $5.84 \pm 0.40$ & $6.01 \pm 1.24$ & $5.98 \pm 0.25$ \\
\hline & 0.185 & $7.62 \pm 0.45^{\mathrm{a}}$ & $8.23 \pm 1.47^{\mathrm{a}}$ & $8.76 \pm 1.98^{\mathrm{a}}$ & $9.33 \pm 0.87^{\mathrm{a}}$ & $11.77 \pm 1.63^{\mathrm{a}}$ \\
\hline & 0.370 & $8.93 \pm 0.68^{\mathrm{a}}$ & $7.52 \pm 1.14^{\mathrm{a}}$ & $9.53 \pm 0.97^{\mathrm{a}}$ & $11.51 \pm 1.15^{\mathrm{a}}$ & $13.67 \pm 2.74^{\mathrm{a}}$ \\
\hline & 0.739 & $9.31 \pm 0.74^{\mathrm{a}}$ & $9.76 \pm 1.03^{\mathrm{a}}$ & $10.77 \pm 1.01^{\mathrm{a}}$ & $13.06 \pm 1.04^{\mathrm{a}}$ & $15.53 \pm 1.95^{\mathrm{a}}$ \\
\hline
\end{tabular}

Values are expressed as mean \pm SE $(n=7)$; MDA: Malondialdehyde level.

${ }^{\mathrm{a}, \mathrm{b}}$ The groups in the same column with different letters are statistically significant $(\mathrm{p}<0.05)$ 
The effects of $\mathrm{CuSO}_{4} .5 \mathrm{H}_{2} \mathrm{O}$ on the $\mathrm{GR}$ activities of the fish tissues are given in Table 2. The results showed that $\mathrm{CuSO}_{4} \cdot 5 \mathrm{H}_{2} \mathrm{O}$ caused a significant decrease in the GR activities the all tissues in a dose-dependent manner. The all group that exposed to $\mathrm{CuSO}_{4} .5 \mathrm{H}_{2} \mathrm{O}$ had no statistically significant effect in a time-dependent manner $(\mathrm{p}<0.05)$.

Table 2. Effect of $\mathrm{CuSO}_{4} .5 \mathrm{H}_{2} \mathrm{O}$ on $\mathrm{GR}$ in gill, kidney and liver of C. umbla (U/mg protein)

\begin{tabular}{|c|c|c|c|c|c|c|}
\hline \multirow{2}{*}{ Indices } & \multirow{2}{*}{ Groups (mg/L) } & \multicolumn{5}{|c|}{ Times (hours) } \\
\hline & & 12 & 24 & 48 & 72 & 96 \\
\hline \multirow[t]{4}{*}{ Gill } & Control & $0.89 \pm 0.11$ & $0.96 \pm 0.07$ & $0.87 \pm 0.09$ & $1.10 \pm 0.13$ & $0.99 \pm 0.11$ \\
\hline & 0.185 & $0.74 \pm 0.21^{\mathrm{a}}$ & $0.77 \pm 0.13^{\mathrm{a}}$ & $0.63 \pm 0.07^{\mathrm{a}}$ & $0.53 \pm 0.22^{\mathrm{a}}$ & $0.59 \pm 0.17^{\mathrm{a}}$ \\
\hline & 0.370 & $0.73 \pm 0.14^{\mathrm{a}}$ & $0.67 \pm 0.07^{\mathrm{a}}$ & $0.40 \pm 0.14^{\mathrm{a}}$ & $0.46 \pm 0.09^{\mathrm{a}}$ & $0.39 \pm 0.10^{\mathrm{a}}$ \\
\hline & 0.739 & $0.61 \pm 0.16^{\mathrm{b}}$ & $0.60 \pm 0.11^{\mathrm{b}}$ & $0.30 \pm 0.03^{b}$ & $0.32 \pm 0.03^{\mathrm{b}}$ & $0.27 \pm 0.16^{\mathrm{b}}$ \\
\hline \multirow[t]{4}{*}{ Kidney } & Control & $1.36 \pm 0.21$ & $1.29 \pm 0.16$ & $1.39 \pm 0.18$ & $1.30 \pm 0.11$ & $1.32 \pm 0.09$ \\
\hline & 0.185 & $1.14 \pm 0.16^{\mathrm{a}}$ & $0.87 \pm 0.21^{\mathrm{a}}$ & $0.77 \pm 0.27^{\mathrm{a}}$ & $0.79 \pm 0.14^{\mathrm{a}}$ & $0.50 \pm 0.11^{\mathrm{a}}$ \\
\hline & 0.370 & $0.92 \pm 0.07^{\mathrm{a}}$ & $0.71 \pm 0.12^{\mathrm{a}}$ & $0.65 \pm 0.09^{\mathrm{a}}$ & $0.64 \pm 0.17^{\mathrm{a}}$ & $0.43 \pm 0.02^{\mathrm{a}}$ \\
\hline & 0.739 & $0.70 \pm 0.10^{\mathrm{b}}$ & $0.60 \pm 0.07^{\mathrm{b}}$ & $0.56 \pm 0.06^{\mathrm{b}}$ & $0.41 \pm 0.03^{b}$ & $0.27 \pm 0.01^{\mathrm{b}}$ \\
\hline \multirow[t]{4}{*}{ Liver } & Control & $1.16 \pm 0.14$ & $1.23 \pm 0.12$ & $1.17 \pm 0.09$ & $1.20 \pm 0.11$ & $1.21 \pm 0.13$ \\
\hline & 0.185 & $0.97 \pm 0.07^{\mathrm{a}}$ & $0.93 \pm 0.27^{\mathrm{a}}$ & $0.89 \pm 0.19^{\mathrm{a}}$ & $0.74 \pm 0.11^{\mathrm{a}}$ & $0.68 \pm 0.18^{\mathrm{a}}$ \\
\hline & 0.370 & $0.76 \pm 0.12^{\mathrm{b}}$ & $0.61 \pm 0.23^{\mathrm{b}}$ & $0.61 \pm 0.21^{\mathrm{b}}$ & $0.43 \pm 0.18^{\mathrm{b}}$ & $0.32 \pm 0.26^{\mathrm{b}}$ \\
\hline & 0.739 & $0.63 \pm 0.04^{\mathrm{b}}$ & $0.47 \pm 0.09^{\mathrm{b}}$ & $0.53 \pm 0.06^{\mathrm{b}}$ & $0.29 \pm 0.12^{\mathrm{b}}$ & $0.21 \pm 0.17^{b}$ \\
\hline
\end{tabular}

Values are expressed as mean $\pm \mathrm{SE}(\mathrm{n}=7)$; GR: Glutathione reductase activity.

${ }^{\mathrm{a}, \mathrm{b}}$ The groups in the same column with different letters are statistically significant $(\mathrm{p}<0.05)$

The tissues G6PD activities showed a statistically $(\mathrm{p}<0.05)$ significant decrease in the groups that were exposed to $\mathrm{CuSO}_{4} .5 \mathrm{H}_{2} \mathrm{O}$ when compared to the control group tissues in a dose-dependent manner. The groups, in a time-dependent manner, had no statistically significant effects (Table 3).

Table 3. Effect of $\mathrm{CuSO}_{4} .5 \mathrm{H}_{2} \mathrm{O}$ on $\mathrm{G} 6 \mathrm{PD}$ in gill, kidney and liver of C. umbla (U/mg protein)

\begin{tabular}{|c|c|c|c|c|c|c|}
\hline \multirow{2}{*}{ Indices } & \multirow{2}{*}{ Groups (mg/L) } & \multicolumn{5}{|c|}{ Times (hours) } \\
\hline & & 12 & 24 & 48 & 72 & 96 \\
\hline \multirow[t]{4}{*}{ Gill } & Control & $0.93 \pm 0.08$ & $0.89 \pm 0.10$ & $0.98 \pm 0.07$ & $1.01 \pm 0.11$ & $0.87 \pm 0.07$ \\
\hline & 0.185 & $0.91 \pm 0.11$ & $0.79 \pm 0.07$ & $0.83 \pm 0.04$ & $0.61 \pm 0.07$ & $0.60 \pm 0.11$ \\
\hline & 0.370 & $0.89 \pm 0.09^{\mathrm{a}}$ & $0.74 \pm 0.04^{\mathrm{a}}$ & $0.70 \pm 0.05^{\mathrm{a}}$ & $0.46 \pm 0.04^{\mathrm{a}}$ & $0.43 \pm 0.05^{\mathrm{a}}$ \\
\hline & 0.739 & $0.74 \pm 0.05^{\mathrm{b}}$ & $0.61 \pm 0.06^{\mathrm{b}}$ & $0.50 \pm 0.07^{\mathrm{b}}$ & $0.36 \pm 0.06^{\mathrm{b}}$ & $0.21 \pm 0.01^{\mathrm{b}}$ \\
\hline \multirow[t]{4}{*}{ Kidney } & Control & $1.49 \pm 0.07$ & $1.53 \pm 0.05$ & $1.50 \pm 0.10$ & $1.57 \pm 0.06$ & $1.53 \pm 0.05$ \\
\hline & 0.185 & $1.03 \pm 0.10^{\mathrm{a}}$ & $0.89 \pm 0.09^{\mathrm{a}}$ & $0.78 \pm 0.07^{\mathrm{a}}$ & $0.66 \pm 0.11^{\mathrm{a}}$ & $0.64 \pm 0.14^{\mathrm{a}}$ \\
\hline & 0.370 & $0.93 \pm 0.17^{\mathrm{a}}$ & $0.78 \pm 0.17^{\mathrm{a}}$ & $0.63 \pm 0.10^{\mathrm{a}}$ & $0.52 \pm 0.11^{\mathrm{a}}$ & $0.49 \pm 0.09^{\mathrm{a}}$ \\
\hline & 0.739 & $0.79 \pm 0.09^{\mathrm{b}}$ & $0.63 \pm 0.15^{\mathrm{b}}$ & $0.56 \pm 0.04^{b}$ & $0.34 \pm 0.02^{\mathrm{b}}$ & $0.30 \pm 0.01^{\mathrm{b}}$ \\
\hline \multirow[t]{4}{*}{ Liver } & Control & $1.48 \pm 0.10$ & $1.57 \pm 0.09$ & $1.62 \pm 0.11$ & $1.50 \pm 0.14$ & $1.63 \pm 0.10$ \\
\hline & 0.185 & $1.01 \pm 0.11^{\mathrm{a}}$ & $0.90 \pm 0.14^{\mathrm{a}}$ & $0.74 \pm 0.08^{\mathrm{a}}$ & $0.77 \pm 0.11^{\mathrm{a}}$ & $0.51 \pm 0.07^{\mathrm{a}}$ \\
\hline & 0.370 & $0.93 \pm 0.21^{\mathrm{a}}$ & $0.81 \pm 0.13^{\mathrm{a}}$ & $0.76 \pm 0.11^{\mathrm{a}}$ & $0.62 \pm 0.09^{\mathrm{a}}$ & $0.45 \pm 0.12^{\mathrm{a}}$ \\
\hline & 0.739 & $0.71 \pm 0.09^{b}$ & $0.65 \pm 0.07^{\mathrm{b}}$ & $0.42 \pm 0.05^{\mathrm{b}}$ & $0.33 \pm 0.01^{\mathrm{b}}$ & $0.24 \pm 0.02^{\mathrm{b}}$ \\
\hline
\end{tabular}

Values are expressed as mean \pm SE $(n=7)$; G6PD: Glucose 6-phosphate dehydrogenase activity.

${ }^{\mathrm{a}, \mathrm{b}}$ The groups in the same column with different letters are statistically significant $(\mathrm{p}<0.05)$ 
SOD activities in all tissues increased significantly in a dose-dependent manner $(p<0.05)$. There was no significant increase in SOD activities in the gill, kidney and liver of the fish exposed to sublethal concentration of the $\mathrm{CuSO}_{4} .5 \mathrm{H}_{2} \mathrm{O}$ compared with the control in a time-dependent manner (Table 4).

Table 4. Effect of $\mathrm{CuSO}_{4} .5 \mathrm{H}_{2} \mathrm{O}$ on SOD in gill, kidney and liver of C. umbla (U/mg protein)

\begin{tabular}{l|c|c|c|c|c|c}
\hline \multirow{3}{*}{ Indices } & \multirow{2}{*}{ Groups (mg/L) } & \multicolumn{5}{|c}{ Times (hours) } \\
\cline { 2 - 6 } & & 12 & 24 & 48 & 72 & 96 \\
\hline Gill & Control & $2.89 \pm 0.21$ & $3.14 \pm 0.16$ & $2.91 \pm 0.15$ & $2.84 \pm 0.19$ & $3.05 \pm 0.15$ \\
& 0.185 & $3.21 \pm 1.69$ & $3.84 \pm 1.23$ & $4.51 \pm 1.23$ & $5.09 \pm 2.27$ & $6.69 \pm 2.06$ \\
& 0.370 & $4.76 \pm 1.01^{\mathrm{a}}$ & $4.93 \pm 1.36^{\mathrm{a}}$ & $5.73 \pm 1.87^{\mathrm{a}}$ & $6.44 \pm 2.47^{\mathrm{a}}$ & $7.56 \pm 2.09^{\mathrm{a}}$ \\
& 0.739 & $6.41 \pm 1.23^{\mathrm{b}}$ & $7.56 \pm 2.08^{\mathrm{b}}$ & $8.97 \pm 2.15^{\mathrm{b}}$ & $9.53 \pm 2.58^{\mathrm{b}}$ & $11.81 \pm 2.15^{\mathrm{a}}$ \\
& & & & & & \\
Kidney & & & & & \\
& Control & $4.78 \pm 2.11$ & $5.08 \pm 1.37$ & $4.85 \pm 0.18$ & $4.93 \pm 0.18$ & $5.12 \pm 0.24$ \\
& 0.185 & $5.93 \pm 1.65^{\mathrm{a}}$ & $7.03 \pm 1.07^{\mathrm{a}}$ & $8.55 \pm 0.61^{\mathrm{a}}$ & $9.01 \pm 1.52^{\mathrm{a}}$ & $9.46 \pm 1.58^{\mathrm{a}}$ \\
& 0.370 & $8.11 \pm 1.54^{\mathrm{a}}$ & $8.43 \pm 0.97^{\mathrm{a}}$ & $7.98 \pm 0.42^{\mathrm{a}}$ & $9.04 \pm 1.24^{\mathrm{a}}$ & $10.51 \pm 1.87^{\mathrm{a}}$ \\
& 0.739 & $10.46 \pm 1.52^{\mathrm{b}}$ & $11.44 \pm 1.47^{\mathrm{b}}$ & $11.99 \pm 0.68^{\mathrm{b}}$ & $13.02 \pm 2.12^{\mathrm{b}}$ & $13.94 \pm 2.45^{\mathrm{b}}$ \\
& & & & & & \\
& & & & & \\
& Control & $3.32 \pm 0.24$ & $3.27 \pm 0.18$ & $3.09 \pm 0.14$ & $3.46 \pm 0.35$ & $3.11 \pm 0.19$ \\
& 0.185 & $3.06 \pm 0.35^{\mathrm{a}}$ & $3.19 \pm 1.21^{\mathrm{a}}$ & $4.36 \pm 0.96^{\mathrm{a}}$ & $5.96 \pm 0.64^{\mathrm{a}}$ & $6.91 \pm 0.95^{\mathrm{a}}$ \\
& 0.370 & $3.61 \pm 1.07^{\mathrm{b}}$ & $4.16 \pm 1.06^{\mathrm{b}}$ & $6.21 \pm 0.24^{\mathrm{b}}$ & $7.82 \pm 0.32^{\mathrm{b}}$ & $8.14 \pm 0.48^{\mathrm{b}}$ \\
& 0.739 & $5.76 \pm 0.75^{\mathrm{c}}$ & $6.21 \pm 0.90^{\mathrm{c}}$ & $8.27 \pm 1.65^{\mathrm{c}}$ & $9.96 \pm 1.76^{\mathrm{c}}$ & $11.01 \pm 0.98^{\mathrm{c}}$ \\
\hline
\end{tabular}

Values are expressed as mean \pm SE ( $n=7)$; SOD: Superoxide dismutase activity.

${ }^{a, b, c}$ The groups in the same column with different letters are statistically significant $(p<0.05)$

The effects of $\mathrm{CuSO}_{4} .5 \mathrm{H}_{2} \mathrm{O}$ exposures on the CAT activities in control and experimental groups are presented in Table $5 . \mathrm{CuSO}_{4} .5 \mathrm{H}_{2} \mathrm{O}$ exposure resulted in a significant increase in the activities of CAT in gill, kidney and liver when compared with the control group fish. However, no statistically significant differences were found in the all tissues (gill, kidney and liver) CAT activities $(\mathrm{p}<0.05)$.

Table 5. Effect of $\mathrm{CuSO}_{4} .5 \mathrm{H}_{2} \mathrm{O}$ on CAT in gill, kidney and liver of C. umbla (U/mg protein)

\begin{tabular}{|c|c|c|c|c|c|c|}
\hline \multirow{2}{*}{ Indices } & \multirow{2}{*}{ Groups (mg/L) } & \multicolumn{5}{|c|}{ Times (hours) } \\
\hline & & 12 & 24 & 48 & 72 & 96 \\
\hline Gill & $\begin{array}{c}\text { Control } \\
0.185 \\
0.370 \\
0.739\end{array}$ & $\begin{array}{l}6.45 \pm 0.64 \\
6.59 \pm 0.24^{\mathrm{a}} \\
7.02 \pm 1.46^{\mathrm{a}} \\
8.42 \pm 1.97^{\mathrm{b}}\end{array}$ & $\begin{array}{c}6.38 \pm 0.86 \\
7.03 \pm 1.42^{\mathrm{a}} \\
8.96 \pm 1.09^{\mathrm{a}} \\
10.76 \pm 2.04^{\mathrm{b}}\end{array}$ & $\begin{array}{c}6.22 \pm 0.34 \\
7.43 \pm 0.65^{\mathrm{a}} \\
9.81 \pm 2.11^{\mathrm{a}} \\
10.97 \pm 1.76^{\mathrm{b}}\end{array}$ & $\begin{array}{c}6.84 \pm 0.76 \\
9.11 \pm 2.36^{\mathrm{a}} \\
8.46 \pm 1.05^{\mathrm{a}} \\
11.78 \pm 1.75^{\mathrm{b}}\end{array}$ & $\begin{array}{c}6.36 \pm 0.36 \\
8.14 \pm 1.68^{\mathrm{a}} \\
10.36 \pm 1.27^{\mathrm{a}} \\
13.11 \pm 1.98^{\mathrm{b}}\end{array}$ \\
\hline Kidney & $\begin{array}{c}\text { Control } \\
0.185 \\
0.370 \\
0.739\end{array}$ & $\begin{array}{c}6.87 \pm 0.43 \\
9.28 \pm 0.97^{\mathrm{a}} \\
10.17 \pm 1.27^{\mathrm{a}} \\
11.23 \pm 1.05^{\mathrm{a}}\end{array}$ & $\begin{array}{c}7.14 \pm 0.75 \\
10.11 \pm 0.46^{\mathrm{a}} \\
11.74 \pm 1.76^{\mathrm{a}} \\
11.97 \pm 1.08^{\mathrm{a}}\end{array}$ & $\begin{array}{c}6.91 \pm 0.61 \\
9.61 \pm 1.07^{\mathrm{a}} \\
11.97 \pm 1.25^{\mathrm{a}} \\
12.04 \pm 1.12^{\mathrm{a}}\end{array}$ & $\begin{array}{c}7.03 \pm 0.56 \\
12.14 \pm 2.36^{\mathrm{a}} \\
12.54 \pm 1.57^{\mathrm{a}} \\
14.71 \pm 2.53^{\mathrm{a}}\end{array}$ & $\begin{array}{c}7.11 \pm 0.04 \\
13.83 \pm 0.64^{\mathrm{a}} \\
14.53 \pm 0.24^{\mathrm{a}} \\
16.76 \pm 0.54^{\mathrm{a}}\end{array}$ \\
\hline Liver & $\begin{array}{c}\text { Control } \\
0.185 \\
0.370 \\
0.739\end{array}$ & $\begin{array}{c}6.11 \pm 0.60 \\
7.06 \pm 1.08^{\mathrm{a}} \\
8.22 \pm 0.86^{\mathrm{b}} \\
10.01 \pm 1.62^{\mathrm{b}}\end{array}$ & $\begin{array}{c}6.05 \pm 0.54 \\
8.57 \pm 0.89^{\mathrm{a}} \\
10.98 \pm 1.05^{\mathrm{b}} \\
11.16 \pm 1.15^{\mathrm{b}}\end{array}$ & $\begin{array}{c}6.28 \pm 0.81 \\
8.37 \pm 1.24^{\mathrm{a}} \\
10.96 \pm 2.18^{\mathrm{b}} \\
11.21 \pm 1.98^{\mathrm{b}}\end{array}$ & $\begin{array}{c}6.31 \pm 0.59 \\
9.94 \pm 0.92^{\mathrm{a}} \\
11.82 \pm 1.54^{\mathrm{b}} \\
13.27 \pm 2.15^{\mathrm{b}}\end{array}$ & $\begin{array}{c}6.23 \pm 0.71 \\
10.39 \pm 1.54^{\mathrm{a}} \\
12.36 \pm 2.73^{\mathrm{b}} \\
13.96 \pm 1.94^{\mathrm{b}}\end{array}$ \\
\hline
\end{tabular}

Values are expressed as mean $\pm \mathrm{SE}(\mathrm{n}=7)$; CAT: Catalase activity.

${ }^{a, b}$ The groups in the same column with different letters are statistically significant $(p<0.05)$ 
Table 6 shows the effects of the $\mathrm{CuSO}_{4} .5 \mathrm{H}_{2} \mathrm{O}$ on the activities of GPx in tissues (gill, kidney and liver) of $C$. umbla. The exposure to $\mathrm{CuSO}_{4} \cdot 5 \mathrm{H}_{2} \mathrm{O}$ caused a significant decrease in the activities of GPx in all the tissues in a dose-dependent manner. In a time-dependent manner, significant differences were not detected in the gill, kidney and liver GPx activities of all experimental group $(\mathrm{p}<0.05)$.

Table 6. Effect of $\mathrm{CuSO}_{4} .5 \mathrm{H}_{2} \mathrm{O}$ on GPx in gill, kidney and liver of C. umbla (U/mg protein)

\begin{tabular}{|c|c|c|c|c|c|c|}
\hline \multirow{2}{*}{ Indices } & \multirow{2}{*}{ Groups (mg/L) } & \multicolumn{5}{|c|}{ Times (hours) } \\
\hline & & 12 & 24 & 48 & 72 & 96 \\
\hline Gill & $\begin{array}{c}\text { Control } \\
0.185 \\
0.370 \\
0.739\end{array}$ & $\begin{array}{c}1.06 \pm 0.11 \\
0.82 \pm 0.18^{\mathrm{a}} \\
0.63 \pm 0.10^{\mathrm{b}} \\
0.56 \pm 0.08^{\mathrm{b}}\end{array}$ & $\begin{array}{c}0.89 \pm 0.09 \\
0.91 \pm 0.12^{\mathrm{a}} \\
0.54 \pm 0.08^{\mathrm{b}} \\
0.51 \pm 0.05^{\mathrm{b}}\end{array}$ & $\begin{array}{c}1.15 \pm 0.17 \\
0.73 \pm 0.21^{\mathrm{a}} \\
0.42 \pm 0.12^{\mathrm{b}} \\
0.43 \pm 0.09^{\mathrm{b}}\end{array}$ & $\begin{array}{c}1.10 \pm 0.11 \\
0.51 \pm 0.09^{\mathrm{a}} \\
0.42 \pm 0.07^{\mathrm{b}} \\
0.32 \pm 0.02^{\mathrm{b}}\end{array}$ & $\begin{array}{c}0.96 \pm 0.12 \\
0.39 \pm 0.11^{\mathrm{a}} \\
0.21 \pm 0.15^{\mathrm{b}} \\
0.13 \pm 0.04^{\mathrm{b}}\end{array}$ \\
\hline Kidney & $\begin{array}{c}\text { Control } \\
0.185 \\
0.370 \\
0.739\end{array}$ & $\begin{array}{l}0.95 \pm 0.09 \\
1.17 \pm 0.61^{\mathrm{a}} \\
0.83 \pm 0.24^{\mathrm{b}} \\
0.70 \pm 0.17^{\mathrm{b}}\end{array}$ & $\begin{array}{c}0.93 \pm 0.13 \\
0.97 \pm 0.25^{\mathrm{a}} \\
0.71 \pm 0.17^{\mathrm{b}} \\
0.57 \pm 0.07^{\mathrm{b}}\end{array}$ & $\begin{array}{l}1.05 \pm 0.10 \\
0.63 \pm 0.11^{\mathrm{a}} \\
0.44 \pm 0.14^{\mathrm{b}} \\
0.42 \pm 0.07^{\mathrm{b}}\end{array}$ & $\begin{array}{c}0.99 \pm 0.14 \\
0.54 \pm 0.21^{\mathrm{a}} \\
0.31 \pm 0.07^{\mathrm{b}} \\
0.38 \pm 0.04^{\mathrm{b}}\end{array}$ & $\begin{array}{c}1.10 \pm 0.09 \\
0.49 \pm 0.13^{\mathrm{a}} \\
0.37 \pm 0.02^{\mathrm{b}} \\
0.21 \pm 0.01^{\mathrm{b}}\end{array}$ \\
\hline Liver & $\begin{array}{c}\text { Control } \\
0.185 \\
0.370 \\
0.739 \\
\end{array}$ & $\begin{array}{c}1.48 \pm 0.19 \\
1.37 \pm 0.34^{\mathrm{a}} \\
0.92 \pm 0.21^{\mathrm{b}} \\
0.73 \pm 0.11^{\mathrm{b}}\end{array}$ & $\begin{array}{c}1.50 \pm 0.16 \\
0.94 \pm 0.17^{\mathrm{a}} \\
0.82 \pm 0.09^{\mathrm{b}} \\
0.52 \pm 0.05^{\mathrm{b}}\end{array}$ & $\begin{array}{c}1.41 \pm 0.12 \\
0.66 \pm 0.14^{\mathrm{a}} \\
0.52 \pm 0.17^{\mathrm{b}} \\
0.50 \pm 0.09^{\mathrm{b}}\end{array}$ & $\begin{array}{c}1.43 \pm 0.19 \\
0.72 \pm 0.11^{\mathrm{a}} \\
0.53 \pm 0.09^{\mathrm{b}} \\
0.43 \pm 0.10^{\mathrm{b}}\end{array}$ & $\begin{array}{c}1.46 \pm 0.14 \\
0.59 \pm 0.26^{\mathrm{a}} \\
0.40 \pm 0.04^{\mathrm{b}} \\
0.29 \pm 0.01^{\mathrm{b}}\end{array}$ \\
\hline
\end{tabular}

Values are expressed as mean \pm SE $(n=7)$; GPx: Glutathione peroxidase activity.

${ }^{\mathrm{a}, \mathrm{b}}$ The groups in the same column with different letters are statistically significant $(\mathrm{p}<0.05)$

\section{Discussion}

Pesticides may induce oxidative stress, leading to the generation of free radicals and causing lipid peroxidation, and may be the underlying molecular mechanism that gives rise to pesticide induced toxicity. Increased lipid peroxidation and oxidative stress can affect the activities of a number of protective enzymatic and non-enzymatic antioxidants that are known to be sensitive indicators of increased oxidative stress (Yonar and Sakin, 2011). Oxidative damage is usually a consequence of the ability of toxic agents to induce free radical generation within the cells. The elevated production of oxygen and nitrogen based radicals and related non-radical products leads to the oxidation of essential macromolecules including lipids, proteins and DNA (Russel et al., 2010). In this study, the effects of $\mathrm{CuSO}_{4} .5 \mathrm{H}_{2} \mathrm{O}$ on antioxidant enzyme activities and lipid peroxidation levels have been investigated in the gill, liver and kidney of freshwater fish C. umbla (Heckel, 1843).

We observed that MDA levels, SOD and CAT activities in the gill, liver and kidney increased by $\mathrm{CuSO}_{4} .5 \mathrm{H}_{2} \mathrm{O}$ in a dose-dependent manner, while the GR, G6PD and GPx activities decreased. Therefore, $\mathrm{CuSO}_{4} .5 \mathrm{H}_{2} \mathrm{O}$-induced stress in the fishes was characterized by decreased GR, G6PD and GPx enzymes and increased lipid peroxidation, SOD and CAT enzymes. Otherwise, in a time-dependent manner, significant differences were not detected in the gill, liver and kidney lipid peroxidation levels and antioxidant enzymes activities of all experimental groups. The response of the antioxidant system to oxidative stress in various tissues shows differences that are due to tissue-specific antioxidant potentials (Ahmad et al., 2000). In this study, significant differences were similarly found in the antioxidant responses and capacities of gill, liver and gills. Yonar et al. (2016) reported that the tissue differences could be 
due to different rates of free radical generation, differences in susceptibility to oxidative damage or different antioxidant capacities of the tissues. $\mathrm{CuSO}_{4}$-induced inhibition and induction in MDA level and antioxidant enzymes activities have been reported in various studies in different fish species (Varo et al., 2007; Trivedi et al., 2012).

MDA are produced by LPO and considered as indicators of oxidative stress, which results from the free radical damage to membrane components of cells (Amin and Hashem, 2012). Yonar et al. (2016) reported that copper sulphate caused an increase in rainbow trout gill and liver MDA levels. Otherwise, Yan et al. (2015) investigated that the effects of different doses of nitenpyram $(0.6,1.2,2.5$ and $5.0 \mathrm{mg} / \mathrm{L})$ and times of exposure (7, 14, 21 and $28 \mathrm{~d}$ ) on liver MDA level of zebrafish. They reported that nitenpyram caused an increase in liver MDA level of zebrafish in a time and dosedependent manner. Also, a lot of researchers have reported a relation between MDA and [Pesticide]-induced stress in fish (Uner et al., 2001; Oruc et al., 2004; Kavasoglu et al., 2015). In our study, exposure of $C$. umbla to all groups concentration of $\mathrm{CuSO}_{4} .5 \mathrm{H}_{2} \mathrm{O}$ shows a marked increase in MDA in the gill, liver and kidney in a dose-dependent manner.

GR activity functions to maintain the cytosolic concentration of reduced glutathione. The induction of GR activity is a potential biochemical marker of oxidative stress (Cazenave et al., 2006). Stara et al. (2012) reported that no significant differences from control values were found in any group for effects of chronic exposure to simazine on activities of GR in tissues of common carp. In this study, throughout the experimental period no significant change in gill, liver and kidney GR activities in a time-dependent manner, but $\mathrm{CuSO}_{4} .5 \mathrm{H}_{2} \mathrm{O}$ caused a significant decrease in the GR activities the all tissues in a dose-dependent manner. Decreased GR activity may lead to GSH depletion if its loss cannot be compensated for by the synthesis of new glutathione molecules (Zhang et al., 2004; Stara et al., 2012)

G6PD is the first enzyme of the pentose phosphate metabolic pathway which is found in the cytosol and in peroxisomes of cells (Frederiks and Vreeling-Sindelárová, 2001; Beydemir et al., 2003). It catalyzes the conversion of glucose6-phosphate to 6phosphogluconate (Ciftci et al., 2007). The most important function of this enzyme is the production of ribose 5-phosphate and NADPH which are necessary for membrane lipids synthesis, reductive and nucleic acid synthesis (Kuo et al., 2000; Ceyhun et al., 2010). In the present study, the tissues G6PD activities showed a statistically significant decrease in the groups that were exposed to $\mathrm{CuSO} 4.5 \mathrm{H} 2 \mathrm{O}$ when compared to the control group tissues in a dose-dependent manner. The groups, in a time-dependent manner, had no statistically significant effects. This finding agrees with previous study by Topal et al. (2014). Topal et al. (2014) investigated that the effect of different doses of chlorpyrifos $(2.25 \mu \mathrm{g} / \mathrm{L}, 4.5 \mu \mathrm{g} / \mathrm{L}$ and $6.75 \mu \mathrm{g} / \mathrm{L})$ and times of exposure $(24,48,72$ and $96 \mathrm{~h})$ on liver and gill G6PD enzyme activity of rainbow trout. They reported that chlorpyrifos caused a decrease in the liver and gill G6PD enzyme activity of rainbow trout.

SOD is an antioxidant enzyme important in inhibiting oxyradical formation and is used as a biomarker to indicate oxidative stress. SOD catalyzes the transformation of superoxide radicals to $\mathrm{H}_{2} \mathrm{O}_{2}$ and $\mathrm{O}_{2}$, and is the first enzyme to deal with oxyradicals (Zhang et al., 2004). Uner et al. (2001) studied the effects of cypermethrin on antioxidant enzymes in liver and kidney of Oreochromis niloticus and Cyprinus carpio. They documented that cypermethrin significantly increased the SOD activities in liver and kidney of Oreochromis niloticus and Cyprinus carpio, when compared to the controls. Yonar et al. (2014) reported that the SOD activity was significantly increased 
in the liver, kidney and gills of Cyprinus carpio of the groups exposed to malathion. The present results showed the SOD activities were increased in the groups that exposed to $\mathrm{CuSO}_{4} \cdot 5 \mathrm{H}_{2} \mathrm{O}$ in different concentrations.

$\mathrm{CAT}$ is an inducible enzyme that protects the biological system against reactive oxygen species (Oruc et al., 2004). When an oxidative stress takes place, most of the superoxide anions $\left(\mathrm{O}_{2}^{-}\right)$released in the cell are transformed into hydrogen peroxide $\left(\mathrm{H}_{2} \mathrm{O}_{2}\right)$ molecules produced inside the peroxisomes, and are destroyed exclusively by CAT (Babo and Vaseur, 1992). Monteiro et al. (2009) investigated that the effects of methyl parathion on antioxidant enzyme activities of Brycon cephalus liver, white muscle and gills. They reported that methyl parathion significantly increased the CAT activity in all tissues when compared to the control. Another study, Yonar et al. (2014) indicated antioxidant enzymatic effects of pesticide malathion, which is an organophosphate pesticide, in the liver, kidney and gill of Cyprinus carpio. They showed that malathion caused a significantly increase in all tissues CAT activities of Cyprinus carpio. Our finding show C. umbla liver, kidney and gill CAT activities significantly increased after exposure to $\mathrm{CuSO}_{4} .5 \mathrm{H}_{2} \mathrm{O}$ in different concentrations. This increases in these enzyme activities are most likely a response to the increased ROS generation induced by pesticide toxicity (John et al., 2001).

GPx catalyses the reduction of hydrogen peroxide and lipid peroxides and is considered an efficient protective enzyme against lipid peroxidation at the expense of GSH (Sakin et al., 2011; Ural et al., 2015; Yonar et al., 2016). The inhibition of the GPx activity by pesticides and $\mathrm{CuSO}_{4}$ has been reported in various studies in fish species. For example; Yonar et al. (2016) reported that GPx activity in liver and gill of rainbow trout was decreased in the $\mathrm{CuSO}_{4}$-induced groups. Similarly, Trivedi et al. (2012) investigated that effects of toxicity of $\mathrm{CuSO}_{4} .5 \mathrm{H}_{2} \mathrm{O}$ on oxidative stress indicators on liver of gold fish (Carassius auratus). They reported that $\mathrm{CuSO}_{4} .5 \mathrm{H}_{2} \mathrm{O}$ caused a reduce in the GPx activities of gold fish liver. Another study, Sayeed et al. (2003) reported that the exposure to deltamethrin caused a significant decrease in the activity of GPx in Channa punctatus gills. Similarly, a lot of researchers have reported a relation between GPx and [Pesticide]-induced stress in fish (Zhang et al., 2004; Monteiro et al., 2006; Monteiro et al., 2009; Stara et al., 2012). The finding of our study showed that the GPx activities in the gill, liver and kidney of $C$. umbla treated with $\mathrm{CuSO}_{4} .5 \mathrm{H}_{2} \mathrm{O}$ were significantly decreased compared to control group in the dose-dependent manner. However, no significant change occurred in the GPx activities in the tissues of the fish exposed to $\mathrm{CuSO}_{4} \cdot 5 \mathrm{H}_{2} \mathrm{O}$. The reduction could be due to its exhaustion or restriction as a result of the increased production of free radicals. The present results signify that the increase in the MDA levels of the fish exposure to different concentrations of $\mathrm{CuSO}_{4} .5 \mathrm{H}_{2} \mathrm{O}$ may be related to the decrease in the GPx activities.

$\mathrm{CuSO}_{4} .5 \mathrm{H}_{2} \mathrm{O}$ is used as an algaecide and a fungicide in aquaculture and agriculture. This compound are, however, highly toxic to fish. In this study, $\mathrm{CuSO}_{4} .5 \mathrm{H}_{2} \mathrm{O}$, significantly decreased the GR, G6PD and GPx activities, but increased the CAT, SOD activities and MDA levels of $C$. umbla liver, kidney and gill tissue in a dose-dependent manner. However, $\mathrm{CuSO}_{4} .5 \mathrm{H}_{2} \mathrm{O}$ exposure did not cause to changes significantly in enzyme activities and MDA levels of $C$. umbla liver, kidney and gill tissue in a timedependent manner. These results indicate the effect of $\mathrm{CuSO}_{4} .5 \mathrm{H}_{2} \mathrm{O}$ in the depletion of antioxidant mechanisms. Future studies should be carried out to elucidate the underlying mechanisms involved in the long-term toxicity profile of $\mathrm{CuSO}_{4} .5 \mathrm{H}_{2} \mathrm{O}$ in C. umbla. 
Acknowledgements. This work was supported by Bingöl University Scientific Research Projects (BUBAP No: 213-242-2015). Therefore, we are grateful to Bingöl University, Turkey.

\section{REFERENCES}

[1] Aebi, H. (1983): Catalase. - In: Bergmeyer, H. U. (ed.) Methods in Enzymatic Analysis. Academic Press, New York.

[2] Ahmad, I., Hamid, T., Fatima, M., Chand, H. S., Jain, S. K., Athar, M., Raisuddin, S. (2000): Induction of hepatic antioxidants in freshwater catfish (Channa punctatus Bloch) is a biomarker of paper mill effluent exposure. - Biochimica et Biophysica Acta 1519: 37-48.

[3] Amin, K. A., Hashem, K. S. (2012): Deltamethrin-induced oxidative stress and biochemical changes in tissues and blood of catfish (Clarias gariepinus): antioxidant defense and role of alpha-tocopherol. - BMC Veterinary Research 8: 45-53.

[4] Atabati, A., Keykhosravi, A., Askari-Hesni, M., Vatandoost, J., Motamedi, M. (2015). Effects of copper sulfate on gill histopathology of grass carp (Ctenopharyngodon idella). -. Iranian Journal of Ichthyology 2: 35-42.

[5] Babo, S., Vaseur, P. (1992): In vitro effects of thiram on liver antioxidant enzyme activities of rainbow trout. - Aquatic Toxicology 22: 61-68.

[6] Beutler, E. (1971): Red Cell Metabolism Manual of Biochemical Methods. - Academic Press, London, England.

[7] Beutler, E. (1975): Red Cell Metabolism. - In: Beutler E. (ed.) A Manual of Biochemical Methods. Grune Strottan, New York.

[8] Beydemir, S., Gulcin, I., Kufrevioglu, O. I., Ciftci, M. (2003): Glucose 6-phosphate dehydrogenase: in vitro and in vivo effects of dantrolene sodium. - Polish Journal of Pharmacology 55: 787-792.

[9] Carlberg, I., Mannervik, B. (1975): Purification and characterization of the flavoenzyme glutathione reductase from rat liver. - Journal of Biological Chemistry 250: 5475-5480.

[10] Cazenave, J., Bistoni, M. D. A., Pesce, S. F., Wunderlin, D. A. (2006): Differential detoxification and antioxidant response in diverse organs of Corydoras paleatus experimentally exposed to microcystin-RR. - Aquatic Toxicology 76: 1-12.

[11] Ceyhun, S. B., Senturk, M., Ekinci, D., Erdogan, O., Ciltas, A., Kocaman, M. E. (2010): Deltamethrin attenuates antioxidant defense system and induces expression of heat shock protein 70 in rainbow trout. - Comparative Biochemistry and Physiology C-Toxicology and Pharmacology 152: 215-223.

[12] Chen, J. C., Lin, C. H. (2001): Toxicity of copper sulfate for survival, growth, molting and feeding of juveniles of the tiger shrimp, Penaeus monodon. - Aquaculture 192: 55-65.

[13] Ciftci, M., Turkoglu, V., Coban, T. A. (2007): Effects of some drugs on hepatic glucose 6-phosphate dehydrogenase activity in Lake Van fish (Chalcalburnus tarischii Pallas, 1811). - Journal of Hazardous Materials 143: 415-418.

[14] Demir, Y., Oruc, E., Topal, A., (2016). Carbonic anhydrase activity responses and histopathological changes in gill and liver tissues after acute exposure to chromium in brown trout juveniles. - Hacettepe Journal of Biology and Chemistry 44: 515-523.

[15] Droge, W. (2002): Free radicals in the physiological control of cell function. Physiological Reviews 82: 47-95.

[16] Durmaz, H., Sevgiler, Y., Üner, N. (2006): Tissue-specific antioxidative and neurotoxic responses to diazinon in Oreochromis niloticus. - Pesticide Biochemistry and Physiology 84: 215-226.

[17] Finney, D. J. (1971): Probit Analysis. - Cambridge University Press, New York.

[18] Frederiks, W. M., Vreeling-Sindelárová, H. (2001): Localization of glucose-6-phosphate dehydrogenase activity on ribosomes of granular endoplasmic reticulum, in peroxisomes and peripheral cytoplasm of rat liver parenchymal cells. - Histochemical Journal 33: 345-353. 
[19] Heo, G. J. (1997): Antibacterial efficacy and safety of copper sulfate pentahydrate to cultured fish. - Korean Journal of Veterinary Research 37: 203-212.

[20] John, S., Kale, M., Rathore, N., Bhatnagar, D. (2001): Protective effect of vitamin E in dimethoate and malathion induced oxidative stress in rat erythrocytes. - Journal of Nutritional Biochemistry 12: 500-504.

[21] Kavasoglu, M., Sarıoglu, Y., Uysal, K., Dönmez, M., Altıkat, S., Yetek, I., Kuru, H. I. (2015): Effect of sodium cyanide on antioxidant enzyme activities and lipid peroxidation in some tissues of mirror carp (Cyprinus carpio). - Pakistan Journal of Zoology 47: 1777-1782.

[22] Kirici, M., Kirici, M., Demir, Y., Beydemir, S., Atamanalp, M. (2016). The effects of $\mathrm{Al}^{3+}$ and $\mathrm{Hg}^{2+}$ on glucose 6-phosphate dehydrogenase from Capoeta umbla kidney. Applied Ecology And Environmental Research 14: 253-264.

[23] Koyun, M. (2011): First record of Dogielius forceps (Monogenea) on Capoeta umbla (Pisces, Cyprinidae) to Turkey, from Murat River. - Aquaculture, Aquarium, Conservation, Legislation - International Journal of the Bioflux Society 4(4): 469-473.

[24] Kumar, N., Prabhu, P. A. J., Pal, A. K., Remya, S., Aklakur, M., Rana, R. S., Gupta, S., Raman, R. P., Jadhao, S. B. (2011): Anti-oxidative and immuno-hematological status of Tilapia (Oreochromis mossambicus) during acute toxicity test of endosulfan. - Pesticide Biochemistry and Physiology 99: 45-52.

[25] Kuo, W., Lin, J., Tang, T. K. (2000): Human glucose-6-phosphate dehydrogenase (G6PD) gene transforms NIH $3 \mathrm{~T} 3$ cells and induces tumors in nude mice. - International Journal of Cancer 85: 857-864.

[26] Lasiene, K., Straukas, D., Vitkus, A., Juodziukyniene, N. (2016): The influence of copper sulphate pentahydrate $\left(\mathrm{CuSO}_{4} \cdot 5 \mathrm{H}_{2} \mathrm{O}\right)$ on the embryo development in the guppies (Poecilia reticulata). Italian Journal of Animal Science 15: 529-535.

[27] Lushchak, V. I. (2011): Environmentally induced oxidative stress in aquatic animals. Aquatic Toxicology 101: 13-30.

[28] Monteiro, D. A., Almeida, J. A., Rantin, F. T., Kalinin, A. L. (2006): Oxidative stress biomarkers in the freshwater characid fish, Brycon cephalus, exposed to organophosphorus insecticide Folisuper 600 (methyl parathion). Comparative Biochemistry and Physiology C-Toxicology and Pharmacology 143: 141-149.

[29] Monteiro, D. A., Rantin, F. T., Kalinin, A. L. (2009): The effects of selenium on oxidative stress biomarkers in the freshwater characid fish matrinxã, Brycon cephalus (Günther, 1869) exposed to organophosphate insecticide Folisuper 600 BR® (methyl parathion). - Comparative Biochemistry and Physiology C-Toxicology and Pharmacology 149: 40-49.

[30] Nouh, W. G, Selim, A. G. (2013): Toxopathological studies on the effect of formalin and copper sulphate in Tilapia as a commonly used disinfectant in aquaculture. - Journal of Applied Environmental and Biological Sciences 3: 7-20.

[31] Oakes, K. D., Van der Kraak, G. J. (2003): Utility of the TBARS assay in detecting oxidative stress in white sucker (Catostomus commersoni) populations exposed to pulp mill effluent. - Aquatic Toxicology 63: 447-463.

[32] Oruc, E. O., Sevgiler, Y., Uner, N. (2004): Tissue-specific oxidative stress responses in fish exposed to 2,4-D and azinphosmethyl. - Comparative Biochemistry and Physiology C-Toxicology and Pharmacology 137: 43-51.

[33] Park, K., Heo, G. J. (2009): Acute and subacute toxicity of copper sulfate pentahydrate $\left(\mathrm{CuSO}_{4} 5 \mathrm{H}_{2} \mathrm{O}\right)$ in the guppy (Poecilia reticulata). - Journal of Veterinary Medical Science 71: 333-336.

[34] Placer, Z. A., Cushman, L., Johnson, B. C. (1966): Estimation of products of lipid peroxidation (malonyldialdehyde) in biological fluids. Analytical Biochemistry 16: 359-364.

[35] Qu, R., Feng, M., Wang, X., Qin, L., Wang, C., Wang, Z., Wang, L. (2014): Metal accumulation and oxidative stress biomarkers in liver of freshwater fish Carassius auratus following in vivo exposure to waterborne zinc under different $\mathrm{pH}$ values. Aquatic Toxicology 150: 9-16. 
[36] Russel, J. R., Lucien, C. M., Dun-Xian, T. (2010): Nuorotoxins: free radical mechanisms and melatonin protection. - Current Neuropharmacology 8: 194-210.

[37] Sakin, F., Ispir, U, Yonar, S. M., Yonar M. E., Taysi, R. (2011): Effect of short-term cypermethrin exposure on oxidant-antioxidant balance in the whole body of rainbow trout fry (Oncorhynchus mykiss). - Fresenius Environmental Bulletin 20: 2806-2809.

[38] Sayeed, I., Parvez, S., Pandey, S., Bin-Hafeez, B., Haque, R., Raisuddin, S. (2003): Oxidative stress biomarkers of exposure to deltamethrin in freshwater fish, Channa punctatus Bloch. - Ecotoxicology and Environmental Safety 56: 259-301.

[39] Stara, A., Machova, J., Velisek, J. (2012): Effect of chronic exposure to simazine on oxidative stress and antioxidant response in common carp (Cyprinus carpio L.). Environmental Toxicology and Pharmacology 33: 334-343.

[40] Storey, K. B. (1996): Oxidative stress: animal adaptation in nature. - Brazilian Journal of Medical and Biological Research 29: 1715-1733.

[41] Sun, Y., Oberley, L. W., Li, Y. (1988): A simple method for clinical assay of superoxide dismutase. - Clinical Chemistry 34: 497-500.

[42] Topal, A., Atamanalp, M., Oruc, E., Kırıc1, M., Kocaman, M. E. (2014): Apoptotic effects and glucose-6-phosphate dehydrogenase responses in liver and gill tissues of rainbow trout treated with chlorpyrifos. - Tissue and Cell 46: 490-496.

[43] Trivedi, M. H., Sangai, N. P., Renuka, A. (2012): Assessment of toxicity of copper sulphate pentahydrate on oxidative stress indicators on liver of gold fish (Carassius auratus). - Bulletin of Environment, Pharmacology and Life Sciences 1: 52-57.

[44] Uner, N., Oruc, E. O., Canli, M., Sevgiler, Y. (2001): Effects of cypermethrin on antioxidant enzyme activities and lipid peroxidation in liver and kidney of the freshwater fish, Oreochromis niloticus and Cyprinus carpio (L.). - Bulletin of Environmental Contamination and Toxicology 67: 657-664.

[45] Ural, M. S. (2013): Chlorpyrifos-induced changes in oxidant/antioxidant status and haematological parameters of Cyprinus carpio carpio: Ameliorative effect of lycopene. Chemosphere 90: 2059-2064.

[46] Ural, M. S., Yonar, M. E., Yonar S. M. (2015): Protective effect of ellagic acid on oxidative stress and antioxidant status in Cyprinus carpio during malathion exposure. Cellular and Molecular Biology 61: 58-63.

[47] Varo, I., Nunes, B., Amat, F., Torreblanca, A., Guilhermino, L., Navarro, J. C. (2007): Effect of sublethal concentrations of copper sulphate on sea bream Sparus aurata fingerlings. - Aquatic Living Resources 20: 263-270.

[48] Yan, S., Wang, J., Zhu, L., Chen, A. Wang, J. (2015): Toxic effects of nitenpyram on antioxidant enzyme system and DNA in zebrafish (Danio rerio) livers. - Ecotoxicology and Environmental Safety 122: 54-60.

[49] Yancheva, V., Velcheva, I., Stoyanova, S., Georgieva, E. (2016). Histological biomarkers in fish as a tool in ecological risk assessment and monitoring programs: a review. Applied Ecology And Environmental Research 14: 47-75.

[50] Yonar, M. E., Sakin, F. (2011): Ameliorative effect of lycopene on antioxidant status in Cyprinus carpio during pyrethroid deltamethrin exposure. - Pesticide Biochemistry and Physiology 99: 226-231.

[51] Yonar, M. E., Ispir, U., Yonar S. M., Kirici, M. (2016): Effect of copper sulphate on the antioxidant parameters in the rainbow trout fry, Oncorhynchus mykiss. - Cellular and Molecular Biology 62: 55-58.

[52] Yonar, S. M., Ural, M. S., Silici, S., Yonar, M. E. (2014): Malathion-induced changes in the haematological profile, the immune response, and the oxidative/antioxidant status of Cyprinus carpio carpio: Protective role of propolis. - Ecotoxicology and Environmental Safety 102: 202-209.

[53] Zhang, J. F., Shen, H., Wang, X. R., Wu, J. C., Xue, Y. Q. (2004): Effects of chronic exposure of 2,4-dichlorophenol on the antioxidant system in liver of freshwater fish Carassius auratus. - Chemosphere 55: 167-174. 